\title{
Identität und Sprache des Migranten in der Fremde im Werk von Franco Biondi
}

\author{
Maria E. Brunner \\ Pädagogische Hochschule Schwäbisch Gmünd
}

Seen from the point of view of literary-sociological studies, Franco Biondi's works are part of the migrant and foreign literatures which emerged in Germany in the wake of the recruitment of foreign labour starting in the 1950s. This literature - written by authors who are not Germans in the sense of the old German nationality and citizenship legislation, but who live in Germany and have their works published in the German language areawas formerly called 'guest-worker literature'. Then, in the 1980s, it was referred to as a literature of 'shock and stunned silence', and in the 1990s as 'migrant literature' or 'literature of foreign parts'. The theme in Biondi's works is the break with origin and the process of 'coming-to-language' of the identity that is forming through the medium of language in the encounter with the foreign. Immigration for Biondi becomes immigration into a new language.

\section{Einleitung}

Literatursoziologisch gesehen ist Biondis Werk Teil jener Migranten- bzw. Ausländerliteratur, die in Deutschland in Folge der in den 50er Jahren einsetzenden Anwerbung ausländischer Arbeitskräfte entstanden ist. Diese Literatur von in Deutschland schreibenden Autoren, die nicht Deutsche im Sinne des alten Staatsbürgerschaftsrechts sind, aber in Deutschland leben und ihre Werke im deutschen Sprachraum publizieren, hieß früher Gastarbeiterliteratur. In den 80er Jahren war dann von einer Literatur der Betroffenheit die Rede, in den 90er Jahren von Migrantenliteratur oder Literatur der Fremde.

Das Thema in Biondis Werk ist der Bruch mit der Herkunft und der Prozess des Zur-Sprache-Kommens, der sich im Medium der Sprache bildenden Identität - sie entwickelt sich in der Begegnung mit der Fremde. Einwanderung wird für ihn zur Einwanderung in eine neue Sprache:

Wie du eben hörst, schließt die deutsche Sprache, eine Sprache, die gemacht wird, eine Erfahrung aus. Warum sagen wir zum Beispiel nicht: Die Unveremigrantlichen? Weil es komisch klingt! [...] Die Unverwurzelichen. Aber Spaß beiseite. So ergeht es oft Minderheiten - ihre Erfahrung ist sprachlich nicht vorgesehen; und wenn einer wie ich sich daran machen will, geht er in der sich verschließenden Sprache unter. (Biondi 1991: 248)

Daher sagt Dario Binachi in Der Stau: Die kulturelle Mehrheit 
denkt, die deutsche Sprache hat nur eine Version, ihre! Daß die Sprache der kulturellen Minderheit, der sie angehört, den Standard bildet, an dem sich Dario bedingungslos auszurichten hat! Sie hat gar nicht mitgekriegt, daß ich hier längst lebe und meine eigentümliche Sprache habe. (Biondi 2001: 264)

So trägt Biondis Literatur gerade durch ihren ästhetischen Eigensinn ein utopisches Potenzial in sich. Biondi will durch seine literarische Produktion eine ganz spezifische Form der Teilnahme am sozialen und politischen Geschehen Deutschlands dokumentieren, die sich in Form einer Überschreitung der grammatikalischen, semantischen und syntaktischen Normen vollzieht, als Form einer bewussten Reflexion in Gestalt einer "Personeneinwanderung" (Biondi 2001: 65).

Der Bruch mit der Heimat, ihren Traditionen und kulturellen Mustern, die Erfahrung der Fremde, die zum Konflikt zwischen dem Eigenen und dem Fremden wird, charakterisieren Biondis literarische Werke in Inhalt und Form. Diese "neue Art des Gestaltens" (Chiellino 1988: 34) ist es, durch die schließlich die Fremde zur Sprache kommt und die literarische Sprache selbst sich wandelt. Kraft ihrer dialogischen Form öffnet sie sich der Begegnung mit dem Fremden - im Gegensatz zu einer Sprache, die "Zäune um sich hatte, die das fremde Leben nicht entließen" (Biondi 1997: 213). Biondis Konzept einer in sich dialogischen Sprache ist als Modell gelungener Kommunikation zu fassen, als Modell einer möglichen Anerkennung des Fremden. Das heißt für den Fremden auch, sich einer "Versöhnung verweigern, die auf Kosten der Differenz der Kulturen stattfinden sollte" (Chiellino 2001: 169).

Franco Biondi findet in seiner Prosa also zu ganz neuen Formen, Fremdheit und Begegnung mit den/dem Fremden, mit dem Anderssein darzustellen. Biondi selbst betrachtet die Arbeitsemigration als eine Variante des Exils. Und dieses Exil, das man zunächst als Fremde bezeichnen kann, dauert an, denn obwohl Biondi als Literat und Psychotherapeut faktisch ein fester Bestandteil der deutschen Gesellschaft geworden ist, empfindet er seine durch die Arbeitsemigration geprägte Position am Rande der deutschen Gesellschaft nicht nur in sozialer und existenzieller, sondern auch in literarischer Hinsicht immer noch als Exil. Trotz seines dreißigjährigen Aufenthalts in Deutschland scheint die kulturelle Andersartigkeit der Literatur, die Biondi produziert, noch keine angemessene Anerkennung gefunden $\mathrm{zu}$ haben. Biondi ist ein Außenseiter in der deutschen Literaturszene geblieben.

Die Fremde ist bei Biondi kein Problem der Geographie, sondern ein soziokulturelles. Nicht nur die Umwelt der neuen Gesellschaft wird in der Emigration zur Fremde, sondern auch sich selbst erkennt der Emigrant in einem unbekannten Land wie einen durch die Erfahrungen in der Fremde Erstarrten, im "Stau".

Der Gebrauch der deutschen Sprache als Medium seiner Produktion ist Resultat einer ästhetischen Entscheidung Biondis, die das literarische Ziel seiner Produktion kenntlich macht: die Idee einer neuen Sprache. Denn Biondis Schreiben dreht sich um den Versuch, der Fremde/dem Fremden in 
der Sprache zu begegnen und sie dialektisch in Nähe zu verwandeln. In seinem ersten Roman (1991) gelingt Biondi der endgültige "Übergang von Autobiographie zu Literatur" (Chiellino 1995: 361). Biondis literarisches Debüt erfolgte noch in italienischer Sprache. Seine frühen Texte aus den 70er Jahren sind hauptsächlich Gedichte. Als wesentlichsten Schritt in die deutsche Sprache wird man aber seine Gedichtsammlung Nicht nur gastarbeiterdeutsch verstehen müssen, die Biondi 1979 im Selbstverlag publiziert. Im Zentrum steht der schwierige Prozess der sprachlichen Auseinandersetzung mit dem Deutschen. Emigration wird geradezu als Einwanderung in die deutsche Sprache dargestellt. Denn trotz der gelungenen Darstellung brisanter soziokultureller Realitäten dokumentiert Biondis Gedichtband den langwierigen Prozess seines Sprachenwechsels. Er thematisiert in seinen Gedichten vorwiegend die Sprachlosigkeit der Ausländer, indem er die deutsche Sprache zum Experimentierfeld ihrer (sprachlichen) Erfahrungen macht, die dann in einer besonderen Form von Gastarbeiterpidgin zum immer wieder ironisch gebrochenen Ausdruck kommen.

Zwischen 1976 und 1982 erscheinen die deutschsprachigen Erzählbände Passavantis Rückkehr (1982a) und Die Tarantel (1982b), mit denen er erstmals eine größere Öffentlichkeit anspricht. Zwar bleibt Biondi den Themen aus seiner früheren Phase treu und stellt die Auseinandersetzung mit den Alltagserfahrungen eines Migranten in der Bundesrepublik weiterhin ins Zentrum, doch ist auch ein gewisser Perspektivenwechsel zu verzeichnen, eine veränderte Einstellung zu Heimat und Herkunft.

Die Fremde erscheint jetzt als konkreter Erfahrungsbereich individueller und sozio-politischer Erfahrungen in Deutschland. Neben der Unmöglichkeit einer Rückkehr - eines der zentralen Themen der Literatur der Ausländer - beschreibt Biondi, wie sich immigrierte Arbeitnehmer mit dem Alltag in der Fremde auseinandersetzen und ihn in biographischer Hinsicht verarbeiten. Die Protagonisten dieser Erzählungen sind nicht mehr so beziehungslos wie früher, sondern sie sind sich ihres fremdbestimmten Ausländerstatus' bewusst; daher reagieren sie darauf und nehmen Anteil am Leben in ihrer neuen Umgebung. Jede Figur agiert in unterschiedlicher Weise, entwickelt ihre Empfindungen und liefert unterschiedliche Lebensstrategien, um das Ausländerdasein zu meistern. So werden Biondis Erzählungen zu Modellen, zu Konfigurationen des Lebens in der Fremde. Am Fall einer Abschiebung thematisiert Franco Biondi in der Novelle Abschied der zerschellten Jahre (1984) die latente Ausländerfeindlichkeit und die Eskalation von physischer Gewalt. In diesem Prosatext steht mit dem Protagonisten Mamo, einem jugendlichen Ausländer der zweiten Generation, der seine neue Heimat über alles liebt, die Problematik der Abschiebung im Brennpunkt des Interesses. Mamo ist sogar bereit, sich mit Gewalt gegen die drohende Abschiebung zu wehren - er verschanzt sich mit einem Maschinengewehr in seiner Dachwohnung. Und so beginnt er erst dann, als seine Ausweisung nicht mehr aufzuhalten ist, über seinen spezifischen sozialen Status als Ausländer zu reflektieren. 


\section{Die Unversöhnlichen oder im Labyrinth der Herkunft}

In Biondis erstem, 1991 erschienenem Roman Die Unversöhnlichen oder im Labyrinth der Herkunft, bleibt die literarische Auseinandersetzung mit der eigenen Identität und die Suche nach der verlorenen Herkunft das bestimmende Thema. Wie in Passavantis Rückkehr kehrt der Protagonist in Die Unversöhnlichen vorübergehend in die ehemalige Heimat zurück, um dort den "Grundkonflikt, der den Ich-Erzähler in die Fremde geführt hat, an seinem Geburtsort zu fassen" (Chiellino 1995: 369).

Fremde ist die bestimmende Signatur der Existenz Binachis - diese Fremde aber weitet sich aus zur Fremde der Herkunft, die ein "Loch in der Existenz" (Biondi 1991: 10) bildet, einen "Riß in der Erinnerung" (16), der durch Erinnerung seine Heilung finden soll. Diese Erinnerung will nicht "zur Entstellung und zur Nostalgie verdammt" (38) sein, sondern sie versteht sich als befreiende Macht. Der Roman handelt von der Befreiung aus der Herkunft durch die literarische Praxis der Erinnerung: Binachi will "in bestimmte Lebensabschnitte zurückkehren, um zu den Orten heimzukehren, wo ich mich befreien kann" (9). Sein Ziel ist "nach San Martino zu fahren, um meiner Familiengeschichte nachzugehen, sie schriftlich zu bewältigen" (15). Denn im "Loch der Erinnerung" verstecken sich die Gründe für die Flucht in die Fremde: "Die Emigration nach Deutschland war eine Flucht vor etwas Anderem. Ein Etwas, das sich im Labyrinth seiner Paranoia verbarg" (129). Schreiben selbst wird nun in der Überwindung nationaler Beschränkungen in einem dialogischen Prozess zum Ort eines bikulturellen Kulturgedächtnisses:

Es war nicht die Frage, ob ich diesen Roman auf Deutsch oder Italienisch niederschrieb: Das war eine ablenkende Frage. Sprache und Vorstellung waren nur der Ausdruck davon, wie tief ich in die Innen- und Außenwelt eindringen würde. (298)

Der Ich-Erzähler Dario Binachi kehrt an seinen Geburtsort San Martino zurück und führt Interviews mit seinen Familienangehörigen. Der Roman erzählt also nicht den Weg in die Fremde, sondern er setzt mit der Reise in das Herkunftsland des Ich-Erzählers ein. Als Auslöser oder Anlass dafür sieht der Ich-Erzähler Binachi seine "quälende Lust [...], niederzuschreiben, was den Riß in der Erinnerung ausmacht", das "Loch in der Existenz" (16). Dem Erzähler geht es bei der Aneignung seiner (Familien-) Geschichte darum, sich seiner Herkunft zu bemächtigen und so auf simultane Weise und gleichsam mit einem Schlag "die Fremde bewohnbar [zu] machen" (291). Die Bewältigung der Herkunft sieht er als Bedingung der Ankunft in der Fremde, die als solche eine Ankunft auch bei sich selbst wäre. Zyklisch und wie unter Wiederholungszwang kehrt der Erzähler in seine Heimat zurück, die ihn buchstäblich nicht loslässt; es ist das "Unerledigte", das Binachi "jedes Jahr ins Land der Herkunft rief" (20), dergestalt, dass die Herkunft "diktatorisch das Leben" (12) des Erzählers bestimmt und es auf diese Weise fremd werden lässt. Unerledigt ist für ihn die Frage: "Wo komme ich her?" 
(23). Er will das Rätsel der Herkunft lösen, ihr "Vergessen" im "Gedächtnis" durch "Identität" (9) ersetzen und so das "Loch in der Existenz" (12) mit Geschichte neu füllen.

Deswegen sucht der Erzähler dieser "Familiensaga" (355) wie ein Detektiv nach Spuren. Das Dorf seiner Geburt ist "jener anonyme und geografische Ort, der nichts mehr zu tun hatte mit meinen Gefühlen” (307). Diese "Kluft zwischen mir und mir" (38) aber ist eine innerliche Differenz, die nicht erst mit der Migration nach Deutschland in Erscheinung trat. ${ }^{1}$ Denn in der Familie kehrt die Fremde nicht nur wieder, sie hat im familiären Kontext in Wirklichkeit sogar ihren Ursprung. Gegenstand des Romans ist deswegen gar nicht die Fremde der (deutschen) Aufnahmegesellschaft, sondern die Genese der Entfremdung als Prozess einer sozialen und personalen Entfremdung im Geburtsort San Martino. Nicht die fremde Gesellschaft des Gastlandes, sondern die Herkunft wird als entfremdende Macht kenntlich. Schon als Kind hat sich der Erzähler als Fremder gefühlt. Die fehlende emotionale Bindung an seinen Herkunftsort und seine Herkunftssprache erklärt Binachi sich vor allem durch die fehlende Bindung an die Mutter, die er fürchtete. Die Distanz zur Mutter ist auch der eigentliche Grund für den Abschied von der Herkunft wie von der Muttersprache; und so ist die deutsche Sprache für ihn mit dem Gewinn an Autonomie verbunden, genauer noch: Schreiben (in deutscher Sprache) wird zum einzigen Medium, in dem sich auf der Schwelle zwischen den Kulturen noch (Rest-)Identität konstituieren kann.

In Die Unversöhnlichen steht nicht die Migration im Zentrum, sondern die Konsequenzen, die sich aus dem Leben in der Fremde sowohl für die Identität des Ich-Erzählers wie für die literarische Bewältigung der Fremde ergeben. Denn der Kulturwechsel wird ebenso mit der Suche nach einer neuen Identität des Schriftstellers verbunden wie mit der Suche nach einer neuen narrativen Strategie. Erschrieben werden soll eine Biographie, die nicht einfach der Bericht einer vermeintlich unmittelbar gegebenen Realität ist und nur wieder ins Gedächtnis gerufen werden muss. ${ }^{2}$

\section{In deutschen Küchen}

Am Ende des Romans Die Unversöhnlichen verschieben sich die Pole von Fremde und Heimat, von Herkunft und Ankunft. Nicht in Deutschland ist der (in Deutschland schreibende) Erzähler angekommen, sondern in seiner Herkunft, vor der er einst meinte, in die Fremde geflohen und dort angekommen zu sein: "So eine lange Reise über tausende von Zeilen, nur um zu entdecken, daß die Flucht in die Fremde ein Weg in deine Herkunft geworden ist" (Biondi 1991: 352). Die Wege kehren sich um: Die Flucht, die zunächst Entfernung vom Geburtsort und vom Haus der Mutter zu sein schien, wird als Reise in die Herkunft kenntlich. Aber das Ziel dieser Reise ist nicht etwa die Ankunft in der Herkunft, die (Wieder-)Verortung im 
Ursprünglichen, sondern die Einsicht in den unheilbaren Riss in der Identität, in die Begegnung mit der immerwährenden Fremde in sich selbst, die den Erzähler lehrt auch das fremde Land (Deutschland) zu bewohnen. In Biondis zweitem Roman In deutschen Küchen wird das Formgesetz der Kontrastierung zur bestimmenden sprachlichen Figur. ${ }^{3}$

So wird ein Spiel mit dem Eigenen und Fremden in Szene gesetzt, d.h. ein Spiel mit den Erwartungen der Leser, um sie wieder zu enttäuschen und ihre Wahrnehmung in eine neue Richtung zu leiten. Doch öffnen sich dadurch auch neue semantische Potenziale. So wird das Wort Mitleid in seine semantischen Konnotationen aufgefächert und auch die Sprache des Wörterbuchs öffnet sich neuen Einblicken und Erfahrungen:

Das Mit vor dem Leid veränderte meine sinnliche Erfahrung. [...] Vor allem spürte ich, daß ein Mit sich niemals mit dem Leid hätte verschmelzen können, wenn man nicht ein Leid fühlte. Mich machte auch stutzig, daß im Wörterbuch nach Leid Leidenschaft stand, und daß die beiden Wörter mit gleichem Stamm völlig verschiedene Bedeutungen hatten. (30)

Der Held des Romans In deutschen Küchen, der derart von der Sprache in Besitz genommen wird, der nach Worten ringt und giert wie sein asthmatischer Vater nach Luft (30), ist wiederum der aus San Martino stammende Dario Binachi. Schauplatz des Ende der sechziger Jahre spielenden Werks, in dem Handlung und Perspektiven nun transparenter erscheinen (vgl. Reeg 1995: 36), ist der fiktive Ort Hüttenheim, den der Autor in der Nähe von Mainz entstehen lässt, zusammen mit seinen Fabriken und seinen landschaftlichen Besonderheiten. Anders als im ersten Roman, der ja gewissermaßen als Reisebericht konzipiert war, nimmt nun die Arbeitswelt und der Alltag der ausländischen wie deutschen Arbeitskräfte einen bedeutenden Raum ein. Der in diesem Alltag vorherrschende Bruch mit der Herkunft, den der Ich-Erzähler in Die Unversöhnlichen noch heilen wollte, wird schon zu Anfang des Romans In deutschen Küchen in einer Wendung konstatiert, von der noch die Rede sein wird: "Seit dem Zug nach Deutschland war Zeit dahin, und die Briefe der Barfreunde, die mir einzig verbliebene Brücke nach San Martino, zeigten Risse" (Biondi 1997: 37). Die Rückkehr nach San Martino bietet für Dario keine Perspektive mehr.

Im Roman In deutschen Küchen bietet die Küche nicht nur einen "Ausschnitt Deutschlands" (233), sondern "jene Distanz, die notwendig war, die Nähe meiner Familie zu ertragen" (93). Ein zentrales Thema ist die Desillusionierung der Sehnsucht nach Heimat und Herkunft. Denn auch in der Heimat war der Protagonist des Romans In deutschen Küchen schon fremd. Ihm ist die Heimat nicht mehr Gegenstand der Sehnsucht, denn in der Fremde wird auch dem Protagonisten Dario die ihn von Geburt an prägende Fremde bewusst, die sein Leben schon im Land der Herkunft bestimmte. Die starre Antithese zwischen Fremde und Heimat wird so in der Erinnerung aufgebrochen, Dario sieht im Industrierevier deutsche "Häuserzeilen, die mich an die Obdachlosenblocks in San Martino erinnerten" (9). Und in der Küche betrachtet der Fremde die Fremde aus der Nähe - "und ich dachte, daß ich 
von dieser Reling aus Gelegenheit hätte, eine Ecke Deutschlands aus der Nähe zu beschauen" (8).

Aufgrund der Schilderungen des Lebens in Wohnheimen, kleinen Zimmern und der Arbeit in der Bitumenfabrik wird dieser in den 60er Jahren spielende Roman als Bildungsroman kenntlich - als Reise des Dario Binachi in die Welt der deutschen Sprache und Literatur. Die Wortlosigkeit des Helden ist nur die Kehrseite eines Banns, den die neue Sprache über ihn verhängt hat: "Ich fühlte, wie die geheimnisvollen Wörter auf mir lasten, wie sie zur Schraubzwinge geworden waren. Die Last der Wörter mußte ich zersprengen" (31). Dario geht es darum, "in eine Sprache einzuwandern, bis diese sich nicht mehr verweigern konnte" (66). Die Struktur des Romans wird von der Frage bestimmt, ob der Protagonist am Ende "sich verlieren oder auch finden" (244) würde. Der Sinn der Entwicklung wird deswegen vom Ende her deutlich, wenn Binachi konstatiert: die "Fremde war zugänglich geworden, mit Vertrautheit angereichert, und einer Sprache, die mich faszinierte" (156). In diesen Prozess des Zur-Sprache-Kommens ist auch die Liebesbeziehung des Helden zur Tochter seiner Hauswirtin eingeschrieben. Denn ist es zunächst die Sprachlosigkeit, welche die Kommunikation mit anderen Menschen in erheblichem Maße reduziert, so ist es später das "Monster der Frotzelsprache" (51). Binachi ist dabei, "der Leidenschaft für Wortumstellungen und anderen Sprachspielen zu frönen, die mir im Alltag mit Einheimischen Entlastung verschaffte" (93). So baut er sich einen "Schutzwall" durch "sprachlich[e] Feldzüge" (96). Aber schließlich wird es ihm "möglich, die sprachlichen Waffen niederzulegen und mit sanfteren Sprachgeplänkeln eine neue Nähe spüren zu lassen" (173). Diese Nähe tritt an die Stelle der "soldateske[n] Aufhebung der Distanz" (174), an die Stelle eines Konzeptes der Eroberung tritt die Annäherung durch Begegnung, die wechselseitige Anerkennung des Fremden: "Ich erlebte, wie sie mein hinkendes Deutsch annahm, ohne Kampfgebärde, ohne den Drang, mich sprachlich zu zähmen oder in meine Sätze So-sagt-man-nicht zu streuen" (188).

Der Prozess der Einwanderung in die deutsche Sprache ist ein Abschied von der Herkunft. Mit der Nähe zur deutschen Sprache und zur Literatur wächst nicht nur die Entfremdung vom italienischen Umfeld, sondern auch die von seiner Geliebten. Im Zu-sich-selbst-Kommen bleiben "Reste von Fremde" (37).

Zur Sprache aber kommt der Held lesend: sind es zunächst Illustrierte, die er verschlingt, so am Ende die Romane von Stendhal. Er findet sich in der "Welt der Bücher" wieder: inzwischen hatte er "in der Stadt auch Buchhandlungen entdeckt..." (270). Literatur wird zum Medium der Selbstverständigung.

Die Wahrnehmung der Sprache der Anderen korrespondiert mit der Entwicklung der sprachlichen Sensibilität Darios:

Grundsätzlich sprachen alle Einheimischen in Gebrochendeutsch mit uns. Ihren weichteigigen Dialekt vermengten sie mit eingeschrumpften Wörtern. 
Wenn sie mit den Zungen ominöse Sprachverunstaltungen schnalzten, schmunzelten wir ihnen gefällig und dankbar zu, wiederholten ihre Worte wie Musterschüler im Unterricht, sprachen mit ihnen, bemüht um Wohlgefallen, Vincenzo in gestelztem Schriftdeutsch, ich in holprigem Hüttenheimerisch, das wahrscheinlich nicht weniger seltsam war als ihr Gastarbeiterdeutsch. (64)

Das Gastarbeiterdeutsch ist ein Angebot, das die Einheimischen dem Fremden $\mathrm{zu}$ dessen Integration bieten; aber es ist ein Angebot, das dem Fremden die sprachlichen Bedingungen diktiert. Der Begriff Gastarbeiterdeutsch kennzeichnet den für den Fremden im Raum der deutschen Sprache vorgesehenen Ort des Marginalisierten. Das dem Fremden zugewiesene Deutsch schreibt dessen Position als Outsider fest, zementiert damit aber auch seine gesellschaftliche Ohnmacht. Mit seinem Hüttenheimerisch versucht Dario im Gegensatz dazu ein eigenes Idiom zu entwickeln, das sich nicht als Echo der gebrochenen Sprache der Einheimischen bildet, sondern an ihren weichteigigen Dialekt anknüpft. Der Dialekt wird ihm zur Behausung sprachlich vermittelter Identität, die er zunächst an Anderen wahrnimmt: "In ihrem Dialekt war die Wirtin sie selbst, in Gebrochendeutsch tat sie meinen Ohren Gewalt an" (46).

Wie ein Nomade zieht Dario von einem Ort zum anderen, überschreitet ständig Grenzen und erweitert seinen Horizont. Nicht nur sprachlich, sondern auch sozial und kulturell lebt er "in (nicht: zwischen) mehreren Welten”, die gleichsam "aufgefächert” (Amodeo 1996: 51) werden. Der Roman öffnet ein Geflecht der Räume als Schauplatz der Handlung. Es gibt nicht nur realistische Milieuschilderungen, auffallend ist vielmehr auch die strukturelle Vernetzung dieser Orte und ihre Beziehung zum Ich-Erzähler. Diese Räume sind Zwischen-Räume, die Fensterküche mit ihrem Fenster in die Welt, die Türschwelle und der Bahnhof. Die Küche der deutschen Vermieter bildet einen Raum des Übergangs, der wie die Sprache des Textes nicht einheitlich ist, sondern sich aus verschiedenen dialektalen Welten zusammensetzt.

Biondi ordnet jeder Figur seines Romans eine unverwechselbare Sprache und Sprechweise zu:

In dem anneren Zimmer wohnt 'ne spanische Familie, sagte die Frau Mitte Dreißig, mit rötlich gefärbten, gewickelten Haaren, in die Wörter hustend. Ihr fehlten zwei Zähne, und in den Löchern rotierte die Zunge. Hier dagejen e Deutscher mit sei Sohn. [...] Auf ein Runterhandeln der Miete ließ sich Frau Pitz nicht ein. Hier nix Italia, hier Jermania! scherzte sie ernst [...]. Das viil, viil Geld, wiederholte mein Vater, und sie rief: Willste oder willste nit? (8-9)

Es kommt nicht zur Mischung der Räume, zu einem kulturellen wie sprachlichen melting pot, sondern auch zu ihrer Schichtung (vgl. Amodeo 1996: 158). Die polyphone Struktur der Romane Biondis wird zu einem hybriden Ort des Dazwischen. In der Überlagerung der verschiedenen Sprachen bildet sich eine dritte Sprache aus, die von einer hybriden Logik der Dialogizität 
geprägt ist, nicht vom Entweder-Oder, sondern von der Denk- und Auslegungsfigur des Sowohl-als-auch.

\section{Der Stau}

Im Roman Der Stau (2001) ist Dario Binachi wieder einer der Protagonisten - er tritt nun auf als "deutschsprachiger Autor mit fremder Zunge" (Biondi 2001: 119). Der Held aber ist der "Sozialarbeiter aus Leidenschaft" (279) Milù Migró, der von einer seltsamen Leidenschaft besessen ist, die er "Personeneinwanderung" (65) nennt. Nicht die Konflikte aufgrund der Emigration bilden das Thema des Romans, sondern die individuellen Konflikte des Helden. Aber gerade in diesen individuellen Konflikten bleibt das Problem der Fremde lebendig, gleichsam als Koketterie mit dem Fremden, die Ausdruck sozialer Beziehungslosigkeit ist, und wohl auch des Unvermögens, in Beziehungen $\mathrm{zu}$ anderen $\mathrm{zu}$ treten: "ich produzierte nur Wege, um mich fern von jeder Liebe zu halten. Ich lachte und rief: Am besten würde ich mit der Fremde fremdgehen!" (27).

Doch die gesellschaftlichen Handlungsfelder haben sich seit seiner Einwanderung endgültig gewandelt:

Eine alte Frau mit schwarzem Gewand und grauem Kopftuch lief am Fenster vorbei. Ihr Gang war sicher, ein Gesichtsausdruck ohne Zweifel, als ob sie schon immer hierzulande lebte. Ich nahm den Kontrast wahr: Wie unsicher die Emigranten vor rund dreißig Jahren auf den Straßen liefen, wie verängstigt sie vor den Kaufhäusern standen. (39)

Plötzlich werden Erfahrungen der Emigration sogar am Schicksal der deutschen Flüchtlinge aus der Ostzone, der Aussiedler, der Vertriebenen, der Flüchtlinge und der Asylanten aufgezeigt:

Ich kannte keinen Ort, in dem mehr als ein Drittel der Bewohner Eltern vorweisen konnte, die in demselben Ort geboren waren. Es gab in jedem Ort die Zugezogenen aus der Stadt, die Eingewanderten aus der Ostzone, die Vertriebenen und Flüchtlinge und die Aussiedler und die Einwanderer und die Asylanten usw. Irgendwann war jeder ein Ehemaliger. Ein jeder verbarg im nachbarschaftlichen Lächeln den Neid und den Argwohn. Im lieblichen Miteinander knisterte der Gegensatz. (20)

Erfahrungen in der Emigration werden jetzt als Schicksal deutscher Flüchtlinge und asylsuchender Menschen erzählt, keine Einzelfälle, sondern conditio humana des Romans Der Stau.

Der Held bleibt ausgegrenzt: "Deutschland ist eine geschlossene Gesellschaft. Multikulti hin, Multikulti her, mit fünf oder zehn oder fünfzehn Millionen Einwanderern bleiben die Deutschen doch unter sich; wir haben dabei nur eine Funktion als Feigenblatt, als exotische Zutat" (41). Die Situation des Schriftstellers Binachi ist auch die Migròs. Denn findet dieser Anerkennung, so allein in der klischeehaften Vorstellung vom Fremden, um 
ihn desto besser "im Käfig der Exotik, vielleicht der Unterwerfung zu halten" (118). Migrò, der Protagonist des Romans Der Stau, lebt ziemlich einsam in einem Land, das ihn nicht zur Kenntnis nimmt.

Im Gegensatz zur Entfremdung, die er bei seiner Klientel immer wieder entdecken muss, sagt Migró von sich: "Ich fühle mich hier zugehörig, aber ich bin fremd" (158). Doch dieses Fremdsein wird im Roman nicht eigens thematisiert, sondern im Grunde vorausgesetzt. Schauplatz ist die Innenwelt des Helden: "In einem fiktiven Deutschland, das in einem entsteht, wenn man sich in Sprache und Kultur hineinbegibt, ohne direkt in dem Land zu leben" (240). Es geht also bei den beiden Protagonisten um "grundverschiedene Bedürfnisse nach Nähe" (158). Der Wert authentischer Existenz wird durch das Konzept der "Personeneinwanderung" (65), in dem äußerste Nähe zu anderen Personen zugleich äußerste Fremde (ihnen und sich selbst gegenüber) bedeutet, in Frage gestellt: "Die Authentizität - existierte sie überhaupt?" (109). Die Pointe aber ist, dass diese Einverleibung nicht des Anderen, sondern in den Anderen gerade eine authentische Form der Existenz ermöglichen soll. Das Einwandern in andere Personen, die Identifikation mit anderen Personen löst den Stau, es kommt zur "Entstauung [...] durch die Entfaltung meiner Personeneinwanderungen" (144), die als Schutz vor "Selbstaufgabe" (220) zu verstehen sind: "Meine Erfüllung enthielt den Drang, in die Individualität anderer Menschen einzuwandern und die Varianten meiner Einwanderung auszuleben, um wirklich hier und jetzt zu sein, ein für allemal" (75).

Die kulturelle Mehrheit, so der Erzähler,

denkt, die deutsche Sprache hat nur eine Version, ihre! Daß die Sprache der kulturellen Minderheit, der sie angehört, den Standard bildet, an dem sich Dario bedingungslos auszurichten hat! Sie hat gar nicht mitgekriegt, daß ich hier längst lebe und meine eigentümliche Sprache habe. (264)

Diese eigentümliche Sprache ist durchaus nicht resistent gegen Kommunikation, entsteht aber im Zeichen eines monologischen, vom realen Austausch mit seiner Umwelt isolierten Bewusstseins. Die Koketterie mit der Fremde führt für den Erzähler zum Scheitern realer Liebe: "ich produzierte nur Wege, um mich fern von jeder Liebe zu halten. Ich lachte und rief: Am besten würde ich mit der Fremde fremdgehen" (27). Der Protagonist und Migrant gesteht so die Unfähigkeit ein, "eine dauerhafte Bindung zu einem anderen Menschen zu gestalten" (75). Hat er für den Versuch der Assimilation nur Verachtung übrig, so droht der Erzähler ebenso sein Selbst zu verlieren durch die frei gewählte Isolation und Distanzierung von anderen Migranten 4 sowie von der "Schicksalsgemeinschaft der Eingeborenen" (267). Als ein solcher Selbstverlust kann die reine und beziehungslose Innerlichkeit des Ichs bezeichnet werden.

Genauso wie die wortwörtliche Übersetzung muttersprachlicher Redewendungen ins Deutsche im Roman In deutschen Küchen nicht aufgrund einer Unfähigkeit der Artikulation in der fremden Sprache erfolgt, sondern aus einem tiefsitzenden Misstrauen in die Sprache der Ankunfts- 
gesellschaft heraus, die die Erfahrung des Migranten nicht zur Sprache kommen lässt, scheitert auch die Einfühlung in andere Menschen aufgrund des generell fehlenden Vertrauens in andere Personen, wobei es sich, und das ist entscheidend für den Migró kennzeichnenden Stau in seinem Inneren, nicht nur um spezifische Repräsentanten der kulturellen Mehrheit handelt. Wird das Ich zum Selbst nur durch reale Beziehungen zu Anderen, so sind die Personeneinwanderungen des Erzählers deren Kompensation; im fiktionalen Bereich der Literatur ersetzen sie die wirkliche Begegnung mit dem Anderen durch die Begegnung mit sich selbst. Denn auf den Mangel an realen Beziehungen zur Fremde antwortet Migró mit seiner obsessiv betriebenen "Personeneinwanderung" (65), die seine Person mit Wirklichkeit ausstatten soll: "Meine Erfüllung enthielt den Drang, in die Individualität der anderen Menschen einzuwandern und die Varianten meiner Einwanderung auszuleben, um wirklich hier und jetzt zu sein, ein für allemal" (75).

Am Ende wird Migrò zum Schriftsteller der eigenen Existenz: "Ich ließ mich nieder und legte los: Eins weiß ich sicher: Meinen Namen: Milú Migrò” (269). Aber dieses Zu-sich-selbst-Kommen wird mit romantischer Geste reflektiert und dadurch irrealisiert. Denn auch Dario Binachi war eine Reflexion seiner selbst im Anderen - die Spiegelung seiner selbst als Autor, als der er sich am Ende wieder findet.

\section{Schluss}

Das Zur-Sprache-Kommen des Migranten ist nicht als Assimilation zu verstehen, sondern als Aneignung der fremden Sprache, als ein dialektischer Prozess, in welchem das Eigene zum Ausdruck zu kommen vermag. Biondi macht sich auf die Suche nach einer neuen Sprache, nach einem neuen Idiom, weil keine der beiden Sprachen, weder das Deutsche noch das Italienische, die Fremde richtig zum Ausdruck bringen kann. ${ }^{5}$

Die Sprache muss sich öffnen, damit das bloß Äußere zur Darstellung des Gefühls der Fremde befähigt wird. ${ }^{6}$ So entsteht in der Prosa Biondis eine neue Unmittelbarkeit durch den Prozess der Entfremdung; erst in diesen Varianten können die Menschen als Individuen bei sich selbst sein. ${ }^{7}$

Aber im Medium der Literatur hat der Eigensinn der Idiome nicht das letzte Wort. Denn man kann zwar feststellen, dass "der kulturelle Unterschied zwischen Italien und Deutschland an einer Redewendung festzumachen ist: Bei den Deutschen befinden sich die Leichen im Keller, bei den Italienern die Skelette im Schrank" (Biondi 2001: 263). Aber dass man den Unterschied übersetzen kann, lässt die Sprache zum Ort eines möglichen Dialogs werden. Über den langwierigen Prozess des Schreibens gelingt zumindest eine gewisse Identitätsfindung.

Literatur ist für Biondi der Ort eines offenen Dialogs, so dass sie zu einem Fragment wirklicher Heimat werden kann. Der Autor in Die Unversöhnlichen weiß, dass "ich den Zeilenfluß und die Kontinuität konstruiere, um die Herkunft zu fühlen, um dadurch den Weg entstehen zu las- 
sen, der aus dem organisierten Leid etlicher Lebensgestaltungen der vielen Dario Binachis in mir hinausführte" (Biondi 1997: 156). Eine so versöhnte wie dialogische Sprache, die das Eigene und das Fremde im Austausch auszutarieren weiß und eine sogeartete, von Bindungslosigkeit geprägte Denkfigur und Lebensweise im Da-zwischen, im Dritten Raum, im Zwischenraum (Hárs 2004: 123) ist das Resultat der Werke Biondis. Die Prosa Biondis lebt von einem Prozess unendlicher Differenzierung, in der Phase eines immer neuen Aufschubs der Identitätsfindung.

Ausgehend von der Beobachtung, dass die Begegnung mit der Fremde/dem Fremden eine für Biondis Prosa konstitutive Rolle spielt - dargestellt an der durchgehend gleich bleibenden Figur eines in Deutschland lebenden Italieners, der aus der Ich-Perspektive erzählt - wurde gezeigt, wie Franco Biondi in seiner Prosa der Fremde bzw. dem Fremden begegnet und wie er beide Denk- und Auslegungsfiguren schließlich auf der Inhalts- und Ausdrucksebene literarisch verarbeitet.

Das Aufeinandertreffen zweier Sprachen und Kulturen provoziert in Biondis frühen Werken wie Passavantis Rückkehr und Abschied der zerschellten Jahre eine Dichotomierung (Entweder-Oder-Situation), eine Art Konfrontation der Kulturen auf Grund der Heterogenität der Herkunftskulturen, um dann in Die Unversöhnlichen und In deutschen Küchen in eher emanzipatorische Potenziale überführt zu werden. Diese emanzipatorischen Potenziale bedingen eine Erweiterung der kognitiven und expressiven Möglichkeiten, d.h. beide Kulturen werden nun von Biondis Protagonisten in einer Art Synthese beherrscht (Sowohl-Als-Auch-Situation).

Der Konflikt zwischen den Kulturen gestaltet sich in Biondis Prosa als Opposition gegen Re-Ethnisierung als Auflösung homogener, kohärenter, nationaler kultureller Systeme und Identitäten sowie als Kritik am Eurozentrismus.

Typisch für Biondis Protagonisten ist auch das dauernde Hin und Her zwischen der alten Welt, die sie verlassen und der neuen Welt, in der sie niemals wirklich zu Hause sind. Die Umstände zwingen sie in einem ZwischenRaum der Kulturen zu leben und keiner von beiden jemals richtig anzugehören. Auf Biondis Protagonisten passt daher die räumliche Metapher des Dazwischen: Die Bilder des Nirgends richtig Dazugehörens und der doppelten Zugehörigkeit laufen hier parallel. Biondi verweist mit seinem Konzept der Transkulturalität auf einen dritten Weg zwischen zwei Kulturen, jenseits von Ghettobildung und Integration. Dazwischen steht eine gemäßigte Position der Integration, die kulturelle Eigenheiten nicht aufgeben, sondern mit den neuen Lebensbedingungen vermitteln will. Die Identitätssuche der Figuren Biondis vollzieht sich deshalb in Form einer Reise zwischen Nähe und Ferne im Raum der Kulturen.

Der Fremde gerät aber nicht allein zwischen die Kulturen, sondern auch zwischen die Sprachen. Der Konflikt zwischen der Kultur der Herkunft und der neuen soziokulturellen Umgebung des Migranten ist daher auch der Konflikt zweier Sprachen. Die Sprache ist es dann auch, die sich für den Fremden als verbindendes Glied zwischen dem Ich und der Gemeinschaft 
erweist, will der Fremde seine Historizität in der neuen Umwelt nicht verlieren. So stehen auch Biondis Protagonisten in einem Spannungsverhältnis zwischen Vertrautheit und Fremde; sie erfahren die neue Kultur und Sprache als Fremde, reflektieren die Bindungen an ihr Herkunftsland und übertragen ihre Erfahrungen schließlich in eine neue Sprache.

Biondis Werk dreht sich immer wieder um den Prozess des ZurSprache-Kommens, der sich im Medium der Sprache bildenden Identität des Fremden, um eine Sprache, die das Eigene und das Fremde in der Begegnung auszutarieren weiß; dabei handelt es sich um eine hybride Sprache, die nicht als Assimilation, sondern als Aneignung der fremden Sprache zu verstehen ist, als dialektischer Prozess. Die durch die Fremde geprägte Sprache, in der Biondi seine Prosa schreibt, und die für ihn als wichtigstes Mittel des Ausdrucks der Fremde fungiert, dialogisiert mit seiner Herkunftssprache und lässt die italienische Sprache, auch wenn diese nicht immer explizit auftaucht, als Resonanzboden mitklingen.

Das Erlernen einer und die Begegnung mit einer fremden Sprache wird so nicht nur als Sprachnot, sondern als befreiende Suche nach einer eigenen Ausdrucksweise dargestellt, die neue Facetten der Wahrnehmung eröffnen und $\mathrm{zu}$ poetischen Sprachspielen jenseits der herkömmlichen Wortbedeutungen führen kann. Der Zusammenprall von Fremdem und Eigenem wird vor allem an dem von der deutschen Standardsprache abweichenden Sprachgebrauch deutlich: in der häufigen Verwendung von Termini in der Muttersprache, von deutschen, der italienischen Sprachstruktur nachgebildeten Neologismen, teilweise als wörtliche Übersetzungen, die im Deutschen durch ihren Verfremdungseffekt komisch wirken. Diese aus der Muttersprache des Autors stammenden Wörter oder Wortspiele wirken im deutschen Text buchstäblich wie Fremdkörper und fungieren als Infragestellungen der Verfügungsmacht der Sprache über den Migranten. Durch den Gebrauch von Wörtern aus der eigenen Muttersprache spielt Biondi mit dem Effekt des fremden Worts und erzeugt damit Kraftfelder zwischen Text und Übersetzung um das, was die sprachliche Konvention verdeckt, endlich deutlich hervorzuheben, sodass die Diskrepanz zwischen dem fremden Wort und der Sprache in den Dienst des Ausdrucks der Wahrheit treten kann.

\section{Bibliographie}

\section{Primärliteratur}

Biondi, Franco (1982a). Passavantis Rückkehr. Fischerhude: Verlag Atelier im Bauernhaus.

Biondi, Franco (1982b). Die Tarantel. Fischerhude: Verlag Atelier im Bauernhaus.

Biondi, Franco (1984). Abschied der zerschellten Jahre. Novelle. Kiel: Neuer Malik Verlag.

Biondi, Franco (1985). Passavantis Rückkehr. Erweiterte Ausgabe. München: Deutscher Taschenbuch Verlag. 
Biondi, Franco (1991). Die Unversöhnlichen oder im Labyrinth der Herkunft. Tübingen: Heliopolis Verlag.

Biondi, Franco (1997). In deutschen Küchen. Frankfurt am Main: Brandes und Apsel Verlag.

Biondi, Franco (2001). Der Stau. Frankfurt am Main: Brandes und Apsel Verlag.

\section{Sekundärliteratur}

Amodeo, Immacolata (1996). Die Ästhetik der Differenz im Werk Franco Biondis. Opladen: Westdeutscher Verlag.

Chiellino, Carmine (1988). Die Reise hält an. München: Beck Verlag.

Chiellino, Carmine (1995). Am Ufer der Fremde. Literatur und Arbeitsemigration (1870-1991). Stuttgart/Weimar: Metzler Verlag.

Chiellino, Carmine (2001). Liebe und Interkulturalität. Essays 1988-2000. Tübingen: Stauffenburg Verlag.

Hárs, Endre (2004). "Postkolonialismus - nur Arbeit am Text? Homi K. Bhabhas theoretisches Engagement". Arcadia 39, 121-135.

Reeg, Ulrike (1995). "Zwischen Italien und Deutschland. Die Vermittlung von 'Raum' und 'Zeit' in den neueren Werken italienischer Autorinnen und Autoren in Deutschland". Caroline Lüderssen \& Salvatore A. Sanna (Hrsg.). Letteratura de-centrata. Italienische Autorinnen und Autoren in Deutschland. Frankfurt am Main: Diesterweg, 27-44.

1 Anlass der Emigration war ökonomische und soziale Not, aber auch das kindliche Trauma väterlicher Autorität und emotionaler Distanz zur Mutter (und ihrer Sprache): "Ich war aus Verzweiflung ausgewandert, die Arbeitsverhältnisse verfluchend, ich hatte mein Heimatland gehaßt" (Biondi 1991: 57).

2 "Und ich werde mir meine Mutter vornehmen, meine Geschwister; meine Schwester wird mir ihren Wagen leihen und ich werde die Verwandten der Reihe nach aufsuchen" (Biondi 1991: 16).

3 Einige Beispiele: "Jugendliche, die an den Computern die Zeit, nein: sich selbst totschlugen" (Biondi 1997: 55); "Hoffnungen auf einen Stellvertretersieg an diesem Schlachttag der Arbeit, spürte die verdeckte Luft des unverrichteten Lebens" (64); "Unglücks-Schöpflöffel" (89).

4 "Sie buhlten um Anerkennung und verloren dabei ihre Selbstachtung" (Biondi 2001: 137).

5 "Es war nicht die Frage, ob ich diesen Roman auf Deutsch oder Italienisch niederschrieb: Das war eine ablenkende Frage. Sprache und Vorstellung waren nur der Ausdruck davon, wie tief ich in die Innen- und Außenwelt eindringen würde" (Biondi 1991: 298).

6 "Ich fühlte, wie die geheimnisvollen Wörter auf mir lasten, wie sie zur Schraubzwinge geworden waren. Die Last der Wörter mußte ich zersprengen" (Biondi 1997: $31)$.

7 "In ihrem Dialekt war die Wirtin sie selbst, in Gebrochendeutsch tat sie meinen Ohren Gewalt an" (Biondi 1997: 46). 